\title{
La sexualidad en adolescentes desde la teoría de las representaciones sociales
}

\section{Sexuality in teenagers from the social representations theory}

\author{
Norma Georgina Martell Martínez ${ }^{1}$, Manuel Leonardo Ibarra Espinosa², \\ Georgina Contreras Landgrave ${ }^{2}$ y Esteban Jaime Camacho Ruiz ${ }^{2}$
}

\begin{abstract}
RESUMEN
El objetivo principal de este estudio fue comprender las representaciones sociales de las relaciones sexuales que tienen los adolescentes, el agente socializador más significativo que interviene en la construcción de estos símbolos, y asimismo las diferencias de representaciones entre hombres y mujeres. Se trata de un estudio de caso a partir del paradigma cualitativo, que tiene como categorías principales la familia, amigos, grupos de pares, medios de comunicación y redes sociales. Se llevaron a cabo entrevistas a profundidad de las que se derivaron las subcategorías de afectividad, compromiso, riesgo, placer y embarazo. Los resultados indican que el papel de la familia es determinante en las conductas sexuales de los jóvenes, y que la figura materna resultó tener una función predominante en la comunicación incluso en los hombres, además de que transmite valores e impone límites. Se encontraron diferencias en las representaciones sociales entre los sexos en cuanto a compromiso y afectividad. Se pudo observar que en el esquema cognitivo de los adolescentes no aparece el riesgo de embarazo y que los medios de comunicación han trazado una ruta orientadora en las relaciones entre los individuos.
\end{abstract}

Palabras clave: Adolescentes; Sexualidad; Representaciones sociales; Salud reproductiva; Conducta sexual.

\begin{abstract}
The aim of the present study was to understand the social representations about sexuality in teenagers, exploring the main socializing agent contributing to building the corresponding symbols, and to exploring the differences between representations by men and women. Through the use of qualitative methodology this case study included family, friends, peer groups, mass media and social networks as main categories. In depth interviews led to discerning such subcategories of affectivity, compromise, risk, pleasure and pregnancy. Results indicate that the family plays a determinant role in the youth's sexual behavior with the mother exerting a predominant influence in communication skills, even for the men, as well as imparting values and imposing limits. Men and women differed regarding compromise and affectivity as well as other differences of meaning between genders. The main findings indicate that teenagers' cognitive scheme does not seem to include the perception of risk of pregnancy, and the mass media have probably become a guiding factor in human relationships.
\end{abstract}

Key words: Teenagers; Sexuality; Social representations; Reproductive health; Sexual behavior.

\footnotetext{
${ }^{1}$ Maestría en Sociología de la Salud, Universidad Autónoma del Estado de México Amecameca, Km 2.5 Carretera Amecameca-Ayapango Francisco Sarabia s/n, 56900 Amecameca, Edo. de México, México, tel. (597)978-21-58, correo electrónico: ginamartell7@hotmail.com. Artículo recibido el 5 de abril y aceptado el 22 de mayo de 2017.

${ }^{2}$ Unidad Académica Profesional Nezahualcóyotl, Universidad Autónoma del Estado de México, Av. Bordo de Xochiaca S/N, Benito Juárez, 57000 Nezahualcóyotl, Edo. de México, México, tel. (55)51-12-63-72, exts. 7962, 7924 y 7912, correos electrónicos: leo.ibarra.uaem@gmail.com, gcontreras@uaemex.mx y jaime_camacho_ruiz@hotmail.com.
} 


\section{INTRODUCCIÓN}

$\mathrm{L}$ a adolescencia es una etapa de la vida significativa que determina la manera de conducirse en la vida adulta debido a los cambios físicos, cognitivos y sociales que se experimentan en aquella; de ahí que la sexualidad y la salud reproductiva tengan un considerable impacto social en las conductas de riesgo (Lavielle et al., 2014). La Organización Mundial de la Salud (OMS) (2016) indica que 16 millones de mujeres de entre 15 y 19 años se embarazan, lo que representa aproximadamente $11 \%$ de los partos en todo el mundo, y que se practican cerca de 2.5 millones de abortos peligrosos. Las tasas de nacimientos prematuros, bajo peso al nacer y asfixia son más altas entre los hijos de adolescentes, lo que aumenta el riesgo de defunción o de futuros problemas de salud en el neonato. La misma OMS menciona que $40 \%$ de los nuevos casos de VIH se presentan en adolescentes.

La salud de los adolescentes y jóvenes es un elemento básico para el avance social, económico y político de un país. Los cambios que ocurren desde el punto de vista biológico, psicológico y social hacen que, después de la infancia, sea la adolescencia la etapa más vulnerable del ciclo vital, por lo que debe ser atendida de manera especial (Martín y Reyes, 2003). Lo anterior indica por qué la salud sexual y reproductiva de los adolescentes se ha constituido como una prioridad nacional e internacional.

El Consejo Nacional de Población (CONAPO) (2014) estima que actualmente hay en el país 22.4 millones de mujeres y hombres adolescentes de entre 10 y 19 años de edad, y que solo $37.6 \%$ de ellos utilizó algún método anticonceptivo en su primera relación sexual. Según la Encuesta Nacional de Salud y Nutrición (ENSANUT) (Instituto Nacional de Salud Pública/Secretaría de Salud, 2012), la proporción de la población de 12 a 19 años en el país que ha iniciado su vida sexual pasó de $15 \%$ en 2006 a 23\% en 2012. Como consecuencia de estas tendencias, también el porcentaje de nacimientos entre madres adolescentes aumentó de 15.6 a $18.7 \%$ entre los años 2003 y 2012 (Secretaría de Salud, 2015). Por su parte, la Estrategia Nacional para la Prevención del Embarazo en Adolescentes (ENAPEA) elaborada por el Gobierno de la República (2014) reconoce la necesidad de desarrollar estrategias para la formación integral en educación sexual en las políticas públicas relativas a la salud y educación.

En el desarrollo sexual del adolescente participan factores como el proceso puberal, la aceptación de la imagen corporal, el descubrimiento de las necesidades sexuales, el desarrollo de la personalidad, el aprendizaje de las relaciones sexuales y el establecimiento de un sistema propio de valores sexuales, todo ello mientras el adolescente está sometido a la presión ejercida por su grupo de iguales o a las reacciones de sus padres ante ese desarrollo sexual (Vega, Robledo, García e Izquierdo, 2012). Es por ello que la sexualidad es uno de los ámbitos de la vida social más simbólicos y contiene múltiples dimensiones que rebasan las condiciones biológicas, por lo que es necesario entender que las valoraciones que tienden a ser compartidas socialmente contribuyen a la identidad social de los individuos, la cual puede reforzarse a partir de una representación social (Moscovici, 1976), y esta a su vez mantiene estructuras de pensamiento compartidas, orientadas hacia la normalización de las conductas sexuales de los adolescentes.

En la presente investigación se toma como marco de referencia la teoría de las representaciones sociales, que permite comprender los fenómenos psicosociales de una manera holística, en tanto que tales representaciones constituyen sistemas cognitivos en los que es posible reconocer estereotipos, opiniones, creencias, valores y normas que orientan la conducta (Araya, 2002), por lo que sirven para guiar, interpretar y justificar el comportamiento.

A través de las relaciones interpersonales se intercambian opiniones, se reafirman conceptos e ideas, se debate y se consolida el proceso de construcción. La representación social se construye en este proceso de intercambio, y al mismo tiempo es ella la que orienta el discurso (Morales, 2002). A través de operaciones mentales como la atención, la percepción y la memoria, un objeto es sustituido por un símbolo, que lo hace presente cuando aquel está ausente (Jodelet, 1988); es decir, el objeto queda representado simbólicamente en la mente de los individuos; no es una simple reproducción del objeto sino que implica su transformación y construcción. 
Moscovici (1976) considera que las representaciones sociales se pueden generar ante situaciones que provocan sentimientos de duda, miedo o incertidumbre, y logran clasificar, reorganizar y explicar aquello que es desconocido, no familiar, en algo conocido y familiar por medio de la función de la objetivación y el anclaje (Alvarado y Garrido, 2003).

Las estadísticas muestran que alrededor de las prácticas sexuales giran aspectos que es conveniente revisar desde la psicología y la sociología de la salud porque no únicamente presentan problemas públicos, sino todo un proceso en el que interactúan conocimientos, opiniones, ideas y actitudes en torno de un hecho que es significativo en la vida de cualquier persona, como la relación sexual, que se expresa también en el ámbito del sentido común como un terreno en el que se pueden recolectar evidencias. Debido a que se trata de un espacio donde las interacciones sociales se manifiesten en diferentes formas, son precisamente las representaciones sociales las que pueden ayudar a identificar la predisposición de una población hacia las relaciones sexuales y sus efectos en la vida social (Crespo, 1995).

Debido a lo anterior, los objetivos de la presente investigación fueron comprender las representaciones sociales que tienen los adolescentes de las relaciones sexuales, e identificar los agentes socializadores más importantes que intervienen en la construcción de dichas representaciones. Se trata de un estudio de caso que utilizó el paradigma cualitativo y entrevistas a profundidad, es decir, encuentros cara a cara entre los participantes y el investigador, orientados a analizar la multiplicidad de percepciones, significados, saberes, códigos, reconocimientos, mitos, ideologías y emociones para lograr un entendimiento detallado de sus experiencias y perspectivas puestas en sus propias palabras, para lo cual es fundamental establecer rapport con los informantes antes de enfocar la investigación (Taylor y Bogdan, 1992).

\section{MÉTODO}

\section{Participantes}

Tomando en cuenta los puntos de saturación y densidad (Mayan, 2001), la población sujeta de estudio fueron cuatro adolescentes, estudiantes de bachillerato técnico en Ixtapaluca, Estado de México (México): dos varones de 17 (E2H) y 18 años (E1H), y dos mujeres de 15 (E3M) y 16 (E4M) años, que representan a los estudiantes en el rango de 15 a 18 años de edad.

\section{Procedimiento}

El muestreo fue por conveniencia y según el área de orientación educativa. Las entrevistas se realizaron con el consentimiento previo por parte del padre o la madre y de los propios entrevistados. Para ello, se diseñó una guía de entrevista con tópicos que contenían categorías de análisis resultantes de la revisión teórica. Dicha guía de la entrevista sirve solamente para recordar que se deben hacer preguntas sobre ciertos temas (Taylor y Bogdan, 1992). Aunque las preguntas están ordenadas, los participantes pueden responderlas libremente, a diferencia de un cuestionario de preguntas cerradas en el que las respuestas deben ser elegidas (Mayan, 2001). La pregunta de investigación que se planteó fue: “¿Cuáles son las representaciones sociales que construyen los jóvenes adolescentes respecto a las relaciones sexuales?

Las categorías contenidas en la guía son la familia, la escuela, los medios de comunicación y las redes sociales. La familia, porque es una institución social que transmite valores tradiciones y educación ya que es la principal encargada de la socialización, que permite al adolescente insertarse al mundo social que le rodea; la escuela, porque su función es la socialización en un contexto institucional, y además porque inculca conocimientos, habilidades, actitudes e intereses; los medios de comunicación, porque transmiten contenidos de información, formación y entretenimiento a través de señales o símbolos, y las redes sociales, porque establecen formas de interacción con amigos o grupo de iguales con los cuales se crean vínculos afectivos y con quienes se comparte un espacio determinado (la escuela) y una etapa particular (la adolescencia).

En el diseño se consideraron aspectos tales como relaciones afectivas, prevención, responsabilidad, compromiso, noviazgo, actividad sexual, placer sexual, riesgo, embarazo, vivencias y experiencias. 
Las entrevistas se llevaron a cabo de forma individual en los cuatro casos. Además, se grabaron en sesiones de una hora y luego se transcribieron por cada caso, clasificando la información por categorías y seleccionando el texto para ingresarlo al software NVivo 11.

Los casos representan las unidades de observación, y a partir de las categorías indicadas arriba resultaron las palabras narrativas que se derivaron de las entrevistas según la cantidad de información observada en las mismas.

A partir de la búsqueda de textos, en las entrevistas resultaron los nodos que se muestran más adelante, en los cuales se esquematizan las palabras narrativas que representan temas, conceptos e ideas reales del esquema cognitivo de cada caso. Se obtuvieron en total 37 nodos en forma de árbol, en los que las ramificaciones representan y clasifican palabras a las cuales se les asignaron atributos y variables en función de las características (edad, sexo etc.). También se obtuvieron dos nodos conglomerados subcategorizando los conceptos de afectividad, compromiso, riesgo, placer y embarazo, jerarquizados en orden de mayor a menor, según la cantidad de información obtenida en las entrevistas. Los resultados (nodos) obtenidos en el software fueron analizados para establecer los referentes de los adolescentes en términos de interacción y comunicación de contenidos, representaciones, imaginarios y prácticas sociales relacionados con la sexualidad para vincularlos con la teoría de las representaciones sociales, esto es, para relacionar los sistemas cognitivos, creencias, opiniones y valores que orientan las prácticas sexuales.

\section{RESULTADOS}

A continuación, se presentan los resultados obtenidos en el software NVivo 11 para investigación cualitativa. En la Figura 1 se muestra un ejemplo de nodo (árbol) de la categoría "familia", y en las Figuras 2 y 3 los conglomerados obtenidos por similitud de palabra y similitud de codificación. La información recabada es muy amplia, por lo que se muestran solo algunos fragmentos de las entrevistas según las categorías de familia, medios de comunicación, redes sociales, escuela, amigos, compromiso y afectividad, así como las diferencias entre las representaciones sociales de hombres y mujeres.

Figura 1. Nodo (árbol) de la categoría "medios de comunicación".

Consulta de búsqueda de texto: vista preliminar de resultados

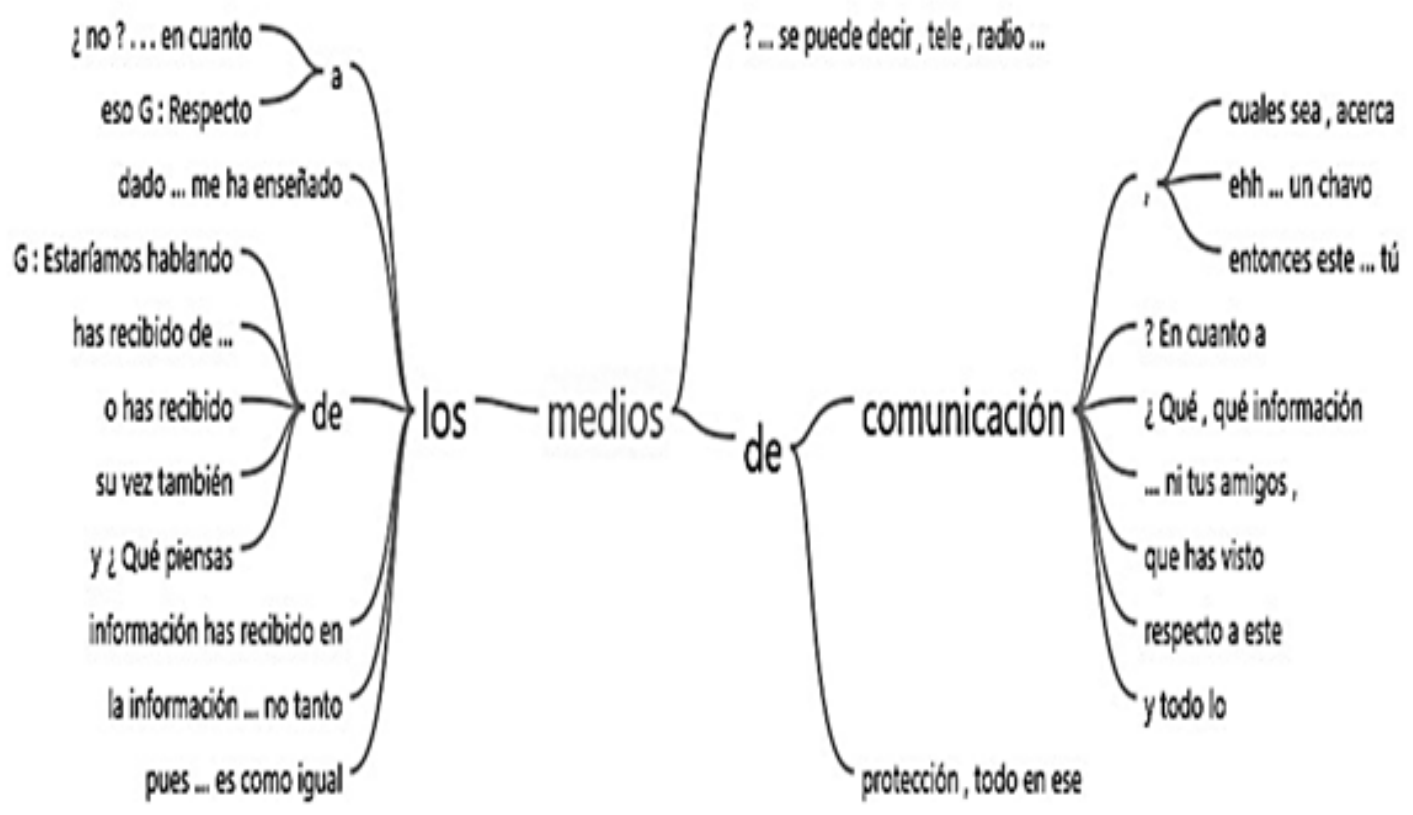


Figura 2. Nodo conglomerado por similitud de palabra.

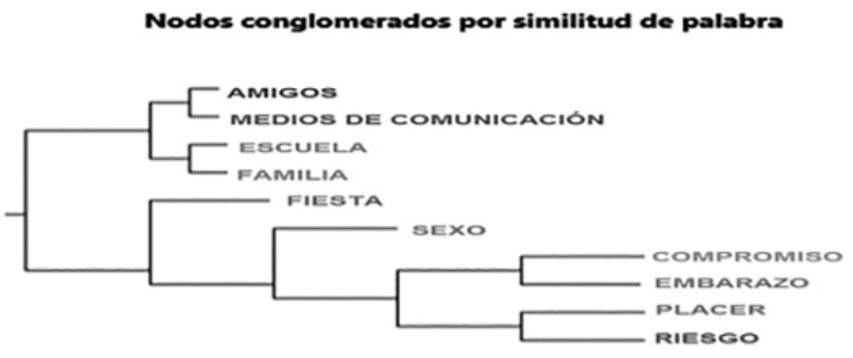

Figura 3. Nodo conglomerado por similitud de codificación.

Nodos conglomerados por similitud de codificación

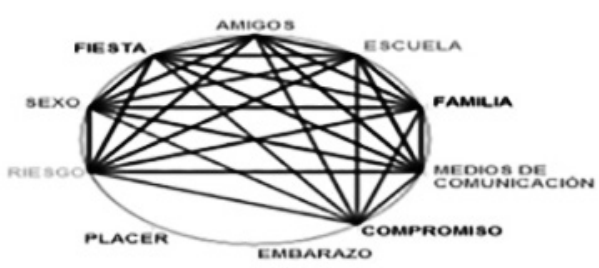

\section{Categoría "Familia"}

En los resultados obtenidos respecto a la categoría "familia", se comprueba que el papel de la familia es determinante en las conductas sexuales de los jóvenes. Moscovici (1976) señala que en el concepto de las representaciones sociales el contexto familiar es el de mayor importancia. En las cuatro entrevistas a profundidad se pone de manifiesto que la familia del adolescente ejerce una importante influencia sobre sus conductas sexuales; es decir, en la relación entre estructura familiar y conductas reproductivas se observan que las formas de comunicación y de interacción familiar tienen gran ascendiente en su conducta sexual y reproductiva, por lo que el contexto familiar puede proveer al adolescente de factores protectores o adversos.

La comunicación en la familia acerca de temas sexuales muestra ser un elemento clave en la construcción de las representaciones. La madre resultó tener un papel predominante en la comunicación, aun entre los varones: "Ella nos habla mucho y nos inculca el cuidado de tener relaciones, las enfermedades, las consecuencias, los embarazos no deseados... todo eso" (E4M).
En dos de los casos se observó que la figura materna transmite valores e impone límites; sin embargo, solo se alude a los métodos de protección. y en uno de ellos, en el que se denota que existía comunicación con ambos padres, la joven no había iniciado aún sus relaciones sexuales: «Bueno, mi familia... Yo siento que sobre ese tema mi mamá ha tenido comunicación conmigo, pero no al cien... Mi mamá me ha dicho "Protégete" y me ha enseñado los medios de protección» $(\mathrm{E} 1 \mathrm{H})$.

\section{Categorías "Medios de comunicación" y "Redes sociales"}

Se observa en el discurso que la publicidad y los medios de comunicación de masas orientan a través de mensajes (eróticos) y que son agentes socializadores potentes en la construcción de las representaciones relativas a las relaciones sexuales de los adolescentes: "La película 50 Sombras de Gray... bueno, yo no he visto la película, pero dicen mis amigos que está padre y que van pasando varias cosas de juegos sexuales" (E2H).

Los resultados muestran cómo los modelos y estereotipos dirigen las formas de comportamiento en los adolescentes: «En Facebook tienden mucho a enviarse fotos o conversaciones, así, desnudas, o fotos desnudas, o que "mándame una foto en calzones" o equis cosa» $(\mathrm{E} 1 \mathrm{H})$.

En los ámbitos social, comunicacional y conversacional, las tecnologías de la información han trazado una ruta orientadora de las relaciones de los individuos: "Entramos en un juego. Armamos un grupo como de ocho personas... Éramos cuatro mujeres y cuatro hombres. Entonces empezamos a jugar Verdad o reto, y entonces nos mandó a tomar una foto en ropa interior" (E3M).

\section{Categorías "Escuela" y "Amigos"}

En los resultados obtenidos respecto a estas categorías, se observa que la interacción con amigos y hermanos influye de manera significativa en el inicio de las relaciones sexuales:

"Ahora la fiesta es para ir a tomar, fumar y ponerse a perrear con cualquier chavo que se junte, y si les da la oportunidad, hasta tienes relaciones sexuales ahí mismo" (E4M). 
"Con los profesores y también con los compañeros de clase, sí hablamos sobre esos temas de sexualidad, o algo así" (E2H).

\section{Subcategorías "Compromiso" y "Afectividad"}

En el nodo conglomerado por similitud de palabra (Figura 2) se muestra la jerarquización según la cantidad de información en los discursos del esquema cognitivo de los jóvenes. En él se observa que la afectividad no se menciona y que no está asociada al compromiso.

Dicha necesidad afectiva no parece ser importante: "Porque también la mujer [...] no necesita nada más estar con un hombre; ella también tiene que experimentar con las demás personas [No debes] esclavizarte a una persona, amarrarte [...]. Tener la libertad de explorar, de probar otras cosas" (E4M). En el imaginario de esta joven, las relaciones prolongadas y con una sola pareja no son deseables, y tener varias relaciones puede convertirse en un elemento de aprendizaje positivo.

En el caso de los hombres, se sorprenden de la falta de compromiso de las mujeres, pero no la cuestionan: "Te dicen así [que] sin compromisos, o sea que nada más quieren tener sexo y no quieren tener nada de compromisos" (E2H).

La reafirmación de la masculinidad en los hombres la define el contexto: «Es solo atracción física y tener [...] ese gusto de decir o presumirles a tus amigos: "Ah, ya me la cogí”, y ellas ya no buscan el mejor hombre de su vida, sino el mejor sexo en su cama» $(\mathrm{E} 1 \mathrm{H})$.

\section{Diferencias entre las representaciones sociales de hombres y mujeres}

Los resultados muestran que hay diferencias en las percepciones, valoraciones e ideologías, así como también en los significados, saberes, códigos, reconocimientos, mitos y emociones entre hombres y mujeres respecto al tema de la sexualidad.

En las mujeres, en los dos casos la representación asocia muy fuertemente las relaciones sexuales con la reproducción y la maternidad: "Pues creo que depende mucho de las definiciones sexuales que se tengan [...]. Se supone que es, biológicamente, para reproducirse, para tener familia, para que siga algo funcionando, las redes humanas: pero igual ya muchos lo toman como juego, como diversión. Ya no son tanto como para lo que se produjo" (E4M).

El sentirse valoradas, amadas y respetadas son los objetivos que más aparecen en el discurso de estas adolescentes: "Bueno, para mí las relaciones son cuando dos personas se quieren mucho, ¿no?, porque para hacer eso no nada más implica tener relaciones y ya nada más por hacerlo, sino debe de ser que las dos personas estén de acuerdo" (E3M).

El siguiente fragmento, de un adolescente varón, pone de manifiesto la desigualdad sexual al momento de interpretar estos procesos: «Rápido, muy fácil... como que ya no le tienes que estar rogando para esas cuestiones [...] Ya si le gustas, pues se avienta a lo que sea, a lo que dé. Y es por eso que las tachan. "Ah, esa chava es bien pronta, o es así, muy loquilla". Y tú ya sabes también a qué le tiras si dices: "No, con esa chava no"» (E2H).

\section{DISCUSIÓN}

Los grupos sociales influyen en la conducta sexual adolescente. Moscovici (1976), en su concepto de las representaciones sociales, señala al contexto familiar como el de mayor importancia entre los demás, lo que se confirma en los presentes resultados. En los cuatro casos se pone de manifiesto que la familia del adolescente tiene una influencia determinante sobre sus conductas sexuales. Los atributos de la familia, como el nivel de comunicación entre sus miembros, el grado de control, el monitoreo de la conducta que lleva a cabo, la calidez y la cercanía de sus miembros desempeñan un importantísimo papel como promotores o protectores de las conductas sexuales de riesgo del adolescente (Lavielle et al., 2014). Sin embargo, esa información es insuficiente, y son los jóvenes que conviven en el seno de familias disfuncionales los más afectados en este aspecto (Domínguez, 2011). En la población estudiada existe una relación que se manifiesta continuamente entre la estructura familiar y sus conductas reproductivas.

Holguín et al. (2013) señalan que en las relaciones entre hijos, padres y hermanos, la madre desempeña un papel importante en la sexualidad del adolescente; tal como se observa en los casos 
incluidos en el presente estudio, es la madre quien ejerce la autoridad en la cognición de las representaciones, misma que se reproduce en la expresión de los jóvenes al mencionar lo que ella les ha enseñado.

En cuanto a los medios de comunicación, el cine proyecta ciertas películas que tratan bajo diferentes perspectivas el embarazo adolescente, $y$ estos textos audiovisuales inciden de manera directa en la reproducción y creación de modelos, actitudes y valores (Marín, 2011). Lo anterior se observa en el discurso de los cuatro participantes, en quienes se comprueba que el papel que desempeñan los medios adquiere sentido en la reproducción de creencias y actitudes. Así, el ambiente que rodea a los adolescentes, formado por la familia, los amigos, los medios de comunicación y las redes sociales, es un factor que influye en la actividad sexual, independientemente de las características sociodemográficas y de los estilos de vida. Ruiz et al. (2012), por ejemplo, consideran que la opinión de los amigos y hermanos sobre el sexo influye en el inicio de las relaciones sexuales entre los jóvenes.

En cuanto a los conceptos de compromiso y afectividad, en la muestra estudiada se evidencia que tener parejas simultáneas evita el compromiso afectivo. En el caso de los hombres, la afirmación de su masculinidad se logra en cierta medida al tener varias relaciones sincrónicas y hablar de ello con sus pares; tal como mencionan Pacheco et al. (2007), ese contexto los impulsa a tener relaciones sexuales como una forma de sustentar su masculinidad. En cuanto a las mujeres, el reconocerse con el derecho de conocer y tener varias relaciones antes de formar una familia, y en los varones tener varias parejas a la vez, es considerado normal. Lo anterior no se aplica sin embargo en el caso de mujeres adolescentes que tienen relaciones simultáneas (Pacheco et al., 2007); en efecto, los adolescentes varones valoran esa conducta en ellos, pero la sancionan en las mujeres.

Es necesario el uso consciente de la comunicación en la enseñanza y el aprendizaje de los deberes y derechos iguales de la mujer y el hombre desde las edades más tempranas como parte de su educación para la sexualidad, lo que puede tener un efecto preventivo en el embarazo adolescente que afecta sobre todo al sexo femenino (Barbón, 2011). Por lo tanto, la dimensión enfocada a las diferencias de género y al sentir de los adolescentes muestran que las representaciones sociales desempeñan un papel fundamental en las prácticas y en las dinámicas de las relaciones sociales, al tiempo que justifican y orientan su conducta.

Respecto a lo anterior, es necesario profundizar en el conocimiento de las representaciones sociales relativas al comportamiento sexual de varones y mujeres, pues es un camino útil para el conocimiento de estos problemas. Ahora bien, el establecimiento de los roles sexuales y sociales define cómo es que hombres y mujeres construyen formas diferentes de ver la sexualidad a través de sus experiencias e interacciones psicológicas y sociales (Moscovici, 1976). Las mujeres relacionan por lo regular la sexualidad con el ejercicio reproductivo y la viven como una experiencia negativa. Entre los varones, no obstante, hay la posibilidad de vivirla positiva y placenteramente (Pacheco et al., 2007). En la muestra investigada se evidencia ese hecho: las mujeres asocian la sexualidad a la reproducción, a diferencia de los hombres, que la refieren como una búsqueda de sensaciones distintas.

La salud de los adolescentes y jóvenes es un asunto que suscita cada vez más interés en todo el mundo. Existe la necesidad de comprender las características de esta edad en el área de la salud pública y las condiciones cambiantes de la sociedad, mismas que, añadidas a la modificación de las conductas de estos grupos, han generado nuevos riesgos para la salud sexual y reproductiva. Por ello, es necesario identificar los valores, creencias y otras cuestiones morales y religiosas que influyen en la decisión de prevenir y proteger a cada persona (Estupiñán, Amaya y Rojas, 2012).

En la presente investigación pudo identificarse que, en el marco interpretativo de los adolescentes, el embarazo y otros riesgos no aparecen en su esquema cognitivo, es decir, no los consideran ni siquiera como una posibilidad, por lo que este problema constituye una preocupación de primer orden para los profesionales de la salud, y se impone abordarlo a partir de un marco conceptual actualizado acerca de un desarrollo humano integral y la promoción de la salud (Martín y Re- 
yes, 2003), pues la conciencia de los mencionados riesgos se genera mediante la prevención y la promoción de la salud sexual y reproductiva.

En las investigaciones sobre la fecundidad y el embarazo adolescente se observa en general que se definen de diversas maneras; se les ve como un problema social e individual en algunos casos, y en otros no se toman en cuenta las diferencias culturales. Por ende, se propone una necesaria reflexión crítica y se esclarezcan las representaciones sociales que tiene el embarazo en distintos estratos socioeconómicos y en ciertas culturas (Gómez et al., 2012).

El contexto sociocultural y económico es esencial en el análisis porque los riesgos para la población adolescente son muy elevados debido a las deprimidas condiciones económicas, la discriminación social y legal, su bajo nivel de instrucción y la falta de oportunidades para su desarrollo integral, todo lo cual constituye un problema de salud pública mundial por el tamaño de esa población (Begazo y Varas, 2015); por consiguiente, las explicaciones en torno a este problema necesitan también integrar el análisis de las condiciones socioeconómicas como uno de los factores determinantes, y entender que no solo es lo social, individual, cultural o económico el origen del problema, sino su compleja interacción.

La conducta sexual de riesgo se estudia por lo regular en instituciones educativas de nivel medio y superior porque frecuentemente el embarazo no deseado entre los estudiantes puede interrumpir su proceso formativo y proyecto de vida (Pulido et al., 2013). Lo anterior trae como consecuencia -entre muchos otros efectos- un aumento del índice de pobreza al no concluir los adolescentes sus estudios, depender de sus padres y no poder insertarse en el mundo laboral. También intervienen elementos socioculturales que se traducen en prácticas sexuales y reproductivas avaladas y reforzadas por el contexto (García, 2016).

Desde esta perspectiva, el embarazo entre los adolescentes amplía las brechas sociales, educativas y de género en cuanto que trasciende el mero proceso biológico reproductivo y afecta proyectos de vida, el ejercicio de libertades y el desarrollo individual, al tiempo que genera conflictos familiares y demandas de atención que requieren de la intervención de diversos agentes sociales.
Por ello, la Estrategia Nacional para la Prevención del Embarazo en Adolescentes (ENAPEA) (Gobierno de la República, 2014) plantea que es necesario ampliar la comprensión del fenómeno para así prevenir su ocurrencia y erradicarlo. Asimismo, la psicología de la salud debe retomar el problema y ofrecer explicaciones atinadas respecto al comportamiento adolescente.

En conclusión, la familia en que conviven los adolescentes es el núcleo que tradicionalmente protege a sus miembros en los aspectos físicos, emocionales y psicológicos y provee los medios para la formación de la personalidad y la transmisión de valores morales y culturales, pero las influencias familiares también ejercen poder en los comportamientos personales de forma negativa. Por lo tanto, la familia puede imponer límites apropiados a la comunicación lesiva y contribuir al establecimiento de conductas sexuales saludables, pero al mismo tiempo puede ser un factor disfuncional que predisponga a situaciones de riesgo por no haber en su seno una guía que oriente el desarrollo de la salud sexual y reproductiva de los asolescentes.

Los medios de comunicación, amigos y grupos de pares mostraron en este estudio ser socializadores potentes de la sexualidad al marcar una orientación específica de las conductas implicadas en aquella. Por otro lado, el embarazo y otros riesgos no se consideran importantes en el esquema cognitivo de los jóvenes; de hecho, no existe en ellos una conciencia clara de los posibles riesgos que podrían evitarse mediante la prevención y promoción de la salud sexual y reproductiva.

Consecuentemente, el análisis contextual es pertinente para entender con mayor profundidad los aspectos socioculturales y socioeconómicos, caracterizados por las desventajas y carencias que enfrenta la población.

El aporte de esta investigación es generar una mayor comprensión de las representaciones sociales de las interacciones sexuales, para ubicar el punto donde se interceptan lo individual y lo social, para apostar así por un cambio de paradigma que propicie nuevas formas de indagación, estrategias y programas de intervención que promuevan la salud sexual; crear una conciencia acerca de los riesgos, consecuencias y responsabilidades individuales y sociales en lo referente a la 
promoción salud sexual y prevención de riesgos, e inducir el desarrollo de los jóvenes a través de la convivencia en espacios recreativos que mejoren su estilo de vida.

\section{REFERENCIAS}

Alvarado, J.L. y Garrido, A. (2003). Psicología social: Perspectivas psicológicas y sociológicas. Madrid: McGraw-Hill.

Araya U., S. (2002). Las representaciones sociales. Ejes teóricos para su discusión. San José de Costa Rica: Facultad Latinoamericana de Ciencias Sociales (FLACSO).

Barbón P., O.G. (2011). Algunas consideraciones sobre comunicación, género y prevención del embarazo adolescente. Ciencia y Enfermería, 17(1), 19-25.

Begazo R., C. y Varas T., W. (2015). La adolescencia como problema de salud reproductiva. Revista Peruana de Ginecología y Obstetricia, 40(2), 33-39.

Consejo Nacional de Población (2014). Salud sexual y reproductiva. México: CONAPO. Disponible en línea: http://www.conapo. gob.mx/es/CONAPO/Salud_Sexual_y_reproductiva (Recuperado el 17 de octubre de 2016).

Crespo, E. (1995). Introducción a la psicología social. Madrid: Editorial Universitaria.

Domínguez, I. (2011). Influencia de la familia en la sexualidad adolescente. Revista Cubana de Obstetricia y Ginecología, 37(3), 387-398.

Estupiñán A., M.R., Amaya E., L.C. y Rojas J., Y.A. (2012). Representaciones sociales de universitarios sobre la abstinencia sexual y los condones como mecanismos de prevención. Revista de Salud Pública, 14(3), 491-501.

García H., G.E. (2016). Mi hijo, lo mejor que me ha pasado en la vida. México: IMJUVE.

Gobierno de la República (2014). Estrategia Nacional para la Prevención del Embarazo en Adolescentes (ENAPEA). México: Autor. Disponible en línea: http://www.issste.gob.mx/images/downloads/home/ENAPEA.pdf (Recuperado el 17 de febrero de 2016).

Gómez S., Á., Gutiérrez M., M.E., Izzedin B., R., Sánchez M., L.M., Herrera M., N.E. y Ballesteros C., M. (2012). Representaciones sociales del embarazo y la maternidad en adolescentes primigestantes y multigestantes en Bogotá. Revista de Salud Pública, 14(2), 189-199.

Holguín Y., P., Mendoza L., A., Esquivel C., M., Sánchez, R., Daraviña A., F. y Acuña, M. (2013). Factores asociados al inicio de la actividad sexual en adolescentes de Tuluá, Colombia. Revista Chilena de Obstetricia y Ginecología, 78(3), 209-219.

Instituto Nacional de Salud Pública y Secretaría de Salud (2012). Encuesta Nacional de Salud y Nutrición (ENSANUT). México: INSP/SS.

Jodelet, D. (1988). La representación social: fenómeno, concepto y teoría. En S. Moscovici (Ed.): Psicología Social II (pp. 469494). Barcelona: Paidós.

Lavielle S., P., Jiménez V., F., Vázquez R., A., Aguirre G., M., Castillo T., M. y Vega M., S. (2014). Impacto de la familia en las conductas sexuales de riesgo de los adolescentes. Revista Médica del IMSS, 52(1), 38-43.

Marín M., F. (2011). Adolescentes y maternidad en el cine: “Juno", "Precious” y “The Greatest”. Comunicar: Revista Cientifica de Comunicación y Educación, 18(36), 115-122.

Martín A., L. y Reyes D., Z. (2003). Conducta sexual, embarazo y aborto en la adolescencia: un enfoque integral de promoción de salud. Revista Cubana de Salud Pública, 29(2), 183-187.

Mayan M., J. (2001). Una introducción a los métodos cualitativos: módulo de entrenamiento para estudiantes y profesores. Alberta (Canadá): Qual Institute Press.

Morales, P. (2002). Psicología social. Buenos Aires: Prentice-Hall.

Moscovici, S. (1976). Psicología Social II. México: Paidós Mexicana.

Organización Mundial de la Salud (2016). Salud de la madre, el recién nacido, del niño y del adolescente. Ginebra: OMS. Disponible en línea: http://www.who.int/maternal_child_adolescent/topics/maternal/adolescent_pregnancy/es/.

Pacheco S., C.I., Rincón S., L.J., Elías G., E., Latorre S., C., Enríquez G., C. y Nieto O., J.M. (2007). Significaciones de la sexualidad y salud reproductiva en adolescentes de Bogotá. Salud Pública de México, 49(1), 45-51.

Pulido R., M.A., Tinoco C., Á., Mondragón J., C.F., Flores L., G., Muñoz L., H. y Campos A., K. R. (2013). Conducta sexual de riesgo en tres universidades privadas de la Ciudad de México. Psicología y Salud, 23(1), 25-32.

Ruiz C., M., López, B., C., Carlos, S., Calatrava, M., Osorio, A. y De Irala, J. (2012). Familia, amigos y otras fuentes de información asociadas al inicio de las relaciones sexuales en adolescentes de El Salvador. Revista Panamericana de Salud Pública, 31, 54-61.

Secretaría de Salud (2015). Salud materna. Disponible en línea http://www.senado.gob.mx/comisiones/igualdad_genero/reu/ docs/presentacion_170615_1.pdf 
Taylor, S.J. Bogdan, R. (1992). Introducción a los métodos cualitativos en investigación. La búsqueda de los significados. Madrid: Paidós.

Vega E., G., Robledo E., M., García P., F. e Izquierdo M., C. (2012). Sexualidad, anticoncepción y conducta sexual de riesgo en adolescentes. International Journal of Psychological Research, 5(1), 79-87. 